\title{
Proteína C reactiva como marcador inflamatorio en la enfermedad periodontal \\ $\mathrm{C}$ reactive protein as an inflammatory marker in periodontal disease
}

Víctor Martínez-Aguilar ${ }^{1,4}$, Bertha Arelly Carrillo-Ávila ${ }^{1}$, Eugenia Guzmán-Marín², Marylín Puerto Solís ${ }^{2}$, Josué R. Bermeo-Escalona ${ }^{3}$ y Amaury Pozos-Guillén ${ }^{4}$

Palabras clave: PCR; enfermedad periodontal; DM2; HbA1c

Recepción: 13-03-17 / Aceptación: 23-05-17

\section{Resumen}

La Proteína C Reactiva (PCR) es una de las proteínas plasmáticas que aparecen en la fase aguda de la inflamación. La periodontitis se relaciona con niveles elevados de PCR en adultos y con una reducción de la misma después de su tratamiento. La diabetes, por otro lado, es una enfermedad que compromete la respuesta tanto inflamatoria como reparativa del organismo y los tejidos periodontales son particularmente sensibles a su efecto. La PCR por lo tanto, puede ser útil en el diagnóstico y en la determinación de progresión de la enfermedad periodontal (EP). El objetivo del presente estudio fue evaluar los niveles de PCR en pacientes con EP y pacientes con EP y diabetes mellitus tipo 2 (DM2). Se incluyeron 60 sujetos distribuidos en 3 grupos: 15 pacientes con EP (Grupo 1), 15 pacientes con EP y DM2 (grupo 2) y 30 pacientes sistémicamente sanos (grupo 3). A cada uno de los participantes se les realizaron pruebas bioquímicas: Proteína C Reactiva (NycoCard® PCR), HbA1c (NycoCard®) y glucosa en sangre. Para el diagnóstico de la enfermedad periodontal se siguieron los parámetros del $5^{\circ}$ Taller Europeo de Periodontología. Para evaluar las diferencias entre los grupos se usó la prueba de Kruskal Wallis. Se encontraron diferencias estadísticas significativas al comparar la concentración de PCR en los 3 grupos $(\mathrm{p}<0.01)$, siendo el grupo de pacientes sanos el que presentaba el menor promedio $(4.88 \pm 0.08)$ y los mayores promedios para los grupos de EP $(5.95 \pm 2.23)$ y EP/DM2 (5.21 \pm 0.20$)$. Además se encontraron diferencias significativas $(\mathrm{p}<0.01)$ en los tres grupos con respecto a la concentración hemoglobina, talla, IMC y PI. Los resultados indican que los niveles séricos de PCR se elevan en pacientes con EP y en pacientes con EP y DM2. Aunque se notó esta diferencia, existen diversos

${ }^{1}$ Facultad de Odontología, Universidad Autónoma de Yucatán. E-mail: vmart81@ gmail.com

${ }^{2}$ Laboratorio de Biología Celular, Centro de Investigaciones Regionales "Dr. Hideyo Noguchi", Universidad Autónoma de Yucatán.

${ }^{3}$ Facultad de Odontología, Universidad De La Salle Bajío.

${ }^{4}$ Doctorado Institucional en Ingeniería y Ciencia de Materiales. Universidad Autónoma de San Luis Potosí.

(C) Universidad De La Salle Bajío (México) 
factores tanto locales como sistémicos que pueden potencialmente influir en los niveles de PCR y estos representan una limitación y dificultad al momento de interpretar las pruebas.

\begin{abstract}
C-Reactive Protein (CRP) is one of the plasma proteins that appear in the acute phase of inflammation. Periodontitis is associated with elevated levels of CRP in adults and with its decreasing after treatment. On the other hand, diabetes is a disease that compromises both the inflammatory and reparative response of the body and the periodontal tissues are particularly sensitive to its effect. Therefore, CRP can be useful for the diagnosis and determination of progression of periodontal disease (PD). The aim of this study was to assess CRP levels in patients with PD and patients with PD and type 2 diabetes (T2D). 60 subjects were included and divided into 3 groups: 15 patients with PD (Group 1), 15 patients with PD and T2D (group 2) and 30 patients systemically healthy (group 3). Each participant underwent biochemical tests: Protein C Reactive (NycoCard $\left.{ }^{\circledR} \mathrm{CPR}\right), \mathrm{HbA1c}\left(\mathrm{NycoCard}{ }^{\circledR}\right)$ and blood glucose test. Weight, height, body mass index (BMI) and periodontal loss of attachment were registered. For diagnosis of periodontal disease, we followed the parameters of the 5th European Workshop on Periodontology. Kruskal Wallis test was used to evaluate the differences between the groups. Significant statistical differences were found when comparing the CRP concentration in the 3 groups $(\mathrm{p}<0.01)$, finding that the healthy group showed the lowest mean $(4.88 \pm 0.08)$ and the highest mean values were shown in the PE groups (5.95 \pm 2.23$)$ and EP/T2D (5.21 \pm 0.20$)$. Also, significant differences $(\mathrm{p}<0.01)$ were found in the three groups, in relation to hemoglobin concentration, height, body mass index and periodontal loss of attachment. The results indicate that serum levels of CRP rise in patients with PD and in patients with PE and T2D. Although this difference was noted, there are several local and systemic factors that can potentially influence CRP levels and these represent a limitation and difficulty when interpreting the tests.
\end{abstract}

Keywords: CRP; Periodontal Disease; T2D. 


\section{Introducción}

La enfermedad periodontal (EF) es un trastorno que afecta las estructuras de inserción del diente, se caracteriza por una exposición bacteriana que puede fomentar una respuesta destructiva del huésped, lo que lleva a la pérdida de inserción periodontal, ósea y por último la posible pérdida de los dientes (Preshaw et al., 2011, 60). De manera genérica hace referencia a dos padecimientos bien diferenciados: la gingivitis y la periodontitis (Wolf et al., 2011, 47). La diferencia entre ambas es que mientras en la gingivitis la afectación se limita a la encía, en la periodontitis se extiende a toda la estructura de soporte e inserción periodontal y en consecuencia, de esta destrucción tisular se puede producir la pérdida dental (Kejri et al., 2014, 56).

La periodontitis en particular, se caracteriza por una inflamación que se extiende hasta la profundidad de los tejidos, y causa la degeneración y destrucción del tejido de soporte y hueso alveolar. El mecanismo fisiopatológico por el cual ocurre este proceso tiene explicación en la respuesta inmune del hospedero frente a los microorganismos productores de toxinas. Éstas endotoxinas estimulan a las células de defensa de los tejidos periodontales a que expresen diferentes mediadores inflamatorios, entre los cuales están la interleucina 1 (IL-1), el factor de necrosis tumoral alfa (FNT- $\alpha$ ) o receptor activador del factor $\kappa \beta$ ligando (RANKL) (Cekici et al., 2014, 57). Sumado a estos mediadores inflamatorios, también se liberan otras sustancias como las proteínas de choque térmico (HSP60) y la Proteína C Reactiva (PCR), entre otras sustancias potencialmente citotóxicas (Bufut et al., 2001, 1). Cuando la respuesta inflamatoria aguda es insuficiente, estas citocinas estimulan a los hepatocitos para que secreten proteínas de fase aguda tales como la PCR durante el proceso de respuesta inflamatoria crónica sistemática no específica (Medzhitov 2007, 819). En sangre, la vida media de la PCR es relativamente corta, apenas 19 horas aproximadamente, y su concentración en un adulto joven con buena salud es de $0.8 \mathrm{mg} / \mathrm{L}$. Sin

embargo, cuando existe algún proceso inflamatorio agudo o una infección bacteriana, la concentración plasmática puede aumentar hasta 10,000 veces (Tsalik et al., 2012, 97).

El nivel sérico de la PCR está elevado a partir de las 6-12 horas de producido un daño tisular, con pico máximo en los 2-3 días posteriores. La producción de PCR no es específica a una enfermedad, pero sus niveles son mayores cuanto es mayor la inflamación y la destrucción tisular.

La periodontitis se relaciona con niveles elevados de PCR en adultos y con una reducción de la misma después de su tratamiento. Se ha propuesto que la PCR puede ser un posible mediador de la asociación entre periodontitis y enfermedades sistémicas inflamatorias (ESI). La diabetes 
mellitus (DM) constituye un grupo de alteraciones metabólicas que se caracteriza por hiperglucemia crónica, debida a un defecto en la secreción de la insulina, a un defecto en la acción de la misma, o a ambas (American Diabetes Association 2010, s62). La diabetes mellitus tipo 2 (DM2) es la forma más frecuente de este padecimiento, en donde existe resistencia insulínica asociada con un déficit real o relativo de insulina. De acuerdo con ENSANUT 2012, la población masculina de 20 años y más, presenta más sobrepeso que obesidad, afecciones que pueden ser detonantes de diabetes. Tanto la EP como la DM son patologías que tienen factores genéticos y alteraciones microbiológicas e inmunológicas en común. La evidencia es que la glucemia se estabiliza después del tratamiento periodontal y la EP produce desestabilización de la glicemia (Peña-Sisto et al., 2008, 1). La EP se convierte en factor de riesgo de la DM, porque en ella la secreción de productos finales de glucosilación avanzados (AGEs), se unen con receptores de membranas de células fagocíticas (monocitos hipersecretores) y sobre regulan las funciones de los mediadores químicos proinflamatorios que mantienen una hiperglucemia crónica, tal y como ocurre en la diabetes (Aramesh et al., 2005, 27). La pérdida de inserción periodontal parece estar estrechamente vinculada al control metabólico de la diabetes. Es así como la presencia de un pobre control de esta enfermedad, medida a través de los niveles plasmáticos de hemoglobina glicosilada (HbA1c), se asocia con mayor prevalencia, severidad y extensión de la EP (Loe 1993, 329).

Otro factor a considerar es el IMC. Estos valores nos indican si el paciente sufre de sobrepeso $(25-<30)$, obesidad ( $>30)$, obesidad grado 1 (30-35), obesidad grado 2 (35-40), grado 3 (>40), y se considera un peso normal cuando el IMC se encuentra en valores entre $18.5-<25$. (Flegal et al., 2013, 71). La asociación entre la inflamación y los rasgos relacionados con la obesidad ha sido estudiada extensamente en los últimos años. Estudios prospectivos reportan asociación entre altos niveles circundantes de PCR y el desarrollo de obesidad y diabetes (Timpson et al., 2011, 300). De igual forma el IMC elevado está relacionado con una alta tasa de mortalidad (obesidad y grado 2 y 3), sin embargo, el sobrepeso está relacionado con una baja de mortalidad. (Flegal et al., 2013, 71). En este contexto, el objetivo del presente estudio fue evaluar los niveles de PCR en pacientes con EP y pacientes con EP y DM2.

\section{Materiales y Métodos}

Se realizó un estudio de casos y controles que incluyó una muestra de 60 pacientes en un rango de edad de 25 a 65 años. La muestra constó de 3 grupos de estudio: el Grupo I fue de 15 pacientes que 
padecían EP; el Grupo II de 15 pacientes con EF y DM2; y el Grupo III de 30 sujetos sistémicamente sanos. Para el Grupo I se seleccionaron pacientes que padecían alguna EP. De acuerdo con los parámetros establecidos por la American Academy of Periodontology 1999 (Kinane 2009, 8), las características clínicas incluyen intensa inflamación gingival, sangrado al sondeo en el área del surco gingival, disminución de la resistencia de los tejidos periodontales al sondeo (bolsa periodontal), pérdida de inserción gingival y del hueso alveolar; además de presentar al menos $30 \%$ de la dentición acometida por la enfermedad y presencia de dientes con nivel de inserción mayor o igual a $5 \mathrm{~mm}$. Los sujetos participantes no debieron haber recibido tratamiento periodontal previo por un periodo de 6 meses. El Grupo II incluyó sujetos que padecían DM2, los cuales fueron confirmados mediante las pruebas bioquímicas realizadas y de acuerdo con los parámetros establecidos por la Asociación Americana de Diabetes (American Diabetes Association 2010, s62). Para el Grupo III, la selección de los participantes se realizó a través de la inclusión de pacientes sin pérdida de inserción periodontal y que no presentaran alteración en los tejidos de sostén del periodonto. Se confirmó que los sujetos de estudio no presentaran algún tipo de enfermedad gingival u oral activa (gingivitis, abscesos periodontales o periapicales, etc.) así como algún tipo de enfermedad sistémica diferente a la DM2 que pudiera afectar los valores de la PCR.

Tras la etapa clínica, se realizó el examen bioquímico que consistió en la toma de muestra de sangre periférica en un horario de 7 a. m. a 11 a. m. y con un ayuno no menor de 8 horas y no mayor de 10 horas, para la determinación de PCR (NycoCard® PCR), HbA1c (NycoCard®) y glucosa en sangre. Además, se tomó el peso, la talla, el IMC y el PI de cada uno de los participantes. No todos los sujetos con DM2 incluidos en el estudio estaban bajo tratamiento médico; algunos de ellos no tenían conocimiento de su padecimiento. Aquellos que manifestaron tener la enfermedad, refirieron estar bajo control médico y refirieron la administración de metformina y glibenclamida. Aquellos sujetos que presentaron niveles elevados de hemoglobina glucosilada y glucosa en sangre fueron referidos con el Médico Internista para confirmación del diagnóstico, y en su caso, control glicémico adecuado. Posteriormente y previo consentimiento, los pacientes fueron tratados en la Clínica de Especialización en Periodoncia. De igual forma, se incluyeron los pacientes con DM2 con y sin control glucémico, ya que en ocasiones los sujetos refirieron tener control adecuado y posteriormente se observó un descontrol del mismo. Para el análisis estadístico se usó el programa SPSS vs. 20 (IBM. Chicago, USA), se realizó estadística descriptiva. Para evaluar las diferencias entre los grupos, se usó la prueba de Kruskal Wallis y se realizó una correlación de Spearman para 
evaluar la relación entre la expresión de PCR y las variables hemoglobina, glucosa, peso, talla, IMC y PI, considerando para ambas pruebas valores de $\mathrm{p} \leq 0.05$ como estadísticamente significativos. Después de realizados los estudios, los participantes recibieron instrucción y motivación hacia la higiene oral y fueron sometidos a profilaxis dentaria. Los participantes del Grupo I con EP fueron referidos para tratamiento periodontal.

\section{Resultados y Discusión}

La mayoría de los participantes fueron del género femenino (60\%). En relación a los controles glucémicos, se registró que el $66.7 \%$ de la población presentaba un buen control de acuerdo con los estudios de hemoglobina glucosilada. No obstante, se encontraron diferencias estadísticas significativas en los 3 grupos con respecto a la concentración de hemoglobina, talla e IMC ( $\mathrm{p}<0.01$; Cuadro 1).

Cuadro 1. Características generales de la muestra.

\begin{tabular}{|c|c|c|c|c|c|c|c|}
\hline \multirow[b]{2}{*}{ GRUPO } & \multicolumn{2}{|c|}{ SEXO } & \multirow{2}{*}{$\begin{array}{c}\text { PESO } \\
\begin{array}{c}\text { Media } \pm D S \\
\text { (Rango) }\end{array}\end{array}$} & \multirow{2}{*}{$\begin{array}{c}\text { TALLA } \\
\text { Media } \pm \text { DS } \\
\text { (Rango) }\end{array}$} & \multirow{2}{*}{$\begin{array}{c}\text { IMC } \\
\begin{array}{c}\text { Media } \pm D S \\
\text { (Rango) }\end{array}\end{array}$} & \multirow{2}{*}{$\begin{array}{c}\text { GS } \\
\text { Media } \pm D \\
\text { S } \\
\text { (Rango) }\end{array}$} & \multirow{2}{*}{$\begin{array}{c}\text { HG } \\
\begin{array}{c}\text { Media } \pm D S \\
(\text { Rango) }\end{array}\end{array}$} \\
\hline & $\begin{array}{c}\text { Mujer } \\
\mathrm{n}=36 \\
(60 \%)\end{array}$ & $\begin{array}{c}\text { Hombre } \\
n=24 \\
(40 \%)\end{array}$ & & & & & \\
\hline $\begin{array}{c}E P \\
\mathbf{n}=\mathbf{1 5}\end{array}$ & $\begin{array}{c}11 \\
(73.3 \%)\end{array}$ & $\begin{array}{c}4 \\
(26.7 \%)\end{array}$ & $\begin{array}{c}79.53 \pm 11.3 \\
(62-97)\end{array}$ & $\begin{array}{l}1.59 \pm 0.58 \\
(1.50-1.7)\end{array}$ & $\begin{array}{l}31.57 \pm 5.07 \\
(24.8-40.6)\end{array}$ & $\begin{array}{c}98.2 \pm 14.7 \\
1 \\
(75-123)\end{array}$ & $\begin{array}{c}5.88 \pm 0.54 \\
(4.8-6.6)\end{array}$ \\
\hline $\begin{array}{c}\text { EP/DM2 } \\
\mathbf{n}=\mathbf{1 5}\end{array}$ & $\begin{array}{c}7 \\
(46.7 \%)\end{array}$ & $\begin{array}{c}8 \\
(53.3 \%)\end{array}$ & $\begin{array}{c}71.20 \pm 9.37 \\
(57-95)\end{array}$ & $\begin{array}{c}1.69 \pm 0.07 \\
(1.60-1.87)\end{array}$ & $\begin{array}{c}24.81 \pm 2.32 \\
(20.23- \\
28.12)\end{array}$ & $\begin{array}{c}113 \pm 28.4 \\
4 \\
(78-158)\end{array}$ & $\begin{array}{c}6.98 \pm 0.65 \\
(5.8-7.9)\end{array}$ \\
\hline $\begin{array}{c}\text { CONTROL } \\
\mathbf{n}=\mathbf{3 0}\end{array}$ & $\begin{array}{c}18 \\
(60 \%)\end{array}$ & $\begin{array}{c}12 \\
(40 \%)\end{array}$ & $\begin{array}{c}71.0 \pm 13.89 \\
(54-96)\end{array}$ & $\begin{array}{c}1.66 \pm 0.08 \\
(1.54-1.82)\end{array}$ & $\begin{array}{c}25.38 \pm 3.35 \\
(21.26- \\
33.22)\end{array}$ & $\begin{array}{c}91.4 \pm 9.03 \\
(80-119)\end{array}$ & $\begin{array}{c}6.0 \pm 0.4 \\
(5.2-6.8)\end{array}$ \\
\hline
\end{tabular}

IMC: Índice de masa corporal GS: Glucosa en sangre HG: Hemoglobina Glucosilada. En cuanto al estado periodontal, se observó una PI de $3.14 \mathrm{~mm}$ en los sujetos con EP, 2.56 en los sujetos con EP/DM2, y $1.16 \mathrm{~mm}$ en los sujetos sanos representando una diferencia significativa entre los grupos $(p<0.01$; Cuadro 2). 
Cuadro 2. Descripción del estado periodontal

\begin{tabular}{ccc}
\hline \multicolumn{2}{c}{ GRUPO } & PI \\
\hline EP & $\mathrm{n}=15$ & $3.14 \pm 0.73(1.2-3.9)$ \\
EP/DM2 & $\mathrm{n}=15$ & $2.56 \pm 0.92(1.2-4.9)$ \\
CONTROL & $\mathrm{n}=30$ & $1.16 \pm 0.18(0.8-1.4)$ \\
\hline
\end{tabular}

PI: Pérdida de inserción.

Al analizar los valores de PCR en los distintos grupos se observó que el grupo con EP reportó 5.9 $\mathrm{mg} / \mathrm{L}$, en tanto el grupo con EP/DM2 presentó $5.2 \mathrm{mg} / \mathrm{L}$ y el grupo de pacientes control $4.88 \mathrm{mg} / \mathrm{L}$ de igual forma observandose una diferencia estadísticamente significativa ( $\mathrm{p}<0.01$; Cuadro 3 ).

Cuadro 3. Valores de Proteína C Reactiva.

\begin{tabular}{ccc}
\hline \multicolumn{2}{c}{ GRUPO } & PCR \\
\hline EP & $\mathrm{n}=15$ & Media \pm DS (Rangos) \\
EP/DM2 & $\mathrm{n}=15$ & $5.9 \pm 2.22(4.90-13.00)$ \\
CONTRO & & \\
$\mathrm{L}$ & $\mathrm{n}=30$ & $4.88 \pm 0.08(4.60-5.00)$ \\
\hline
\end{tabular}

PCR: Proteína $\mathrm{C}$ reactiva. 
Finalmente, mediante la prueba de Spearman encontramos una correlación estadísticamente significativa de la expresión de PCR con el aumento de la hemoglobina ( $R h o=0.384 ; p=0.002)$ y con valores más altos de PI (Rho=0.540; $\mathrm{p}<0.01)$.

A pesar de que existen diversos estudios que sugieren una posible relación entre la inflamación crónica, mediadores proinflamatorios e inmunológicos que provienen de la EP con el aumento de los niveles séricos de PCR; aún no es clara la función que tiene ésta durante la manifestación de estos procesos. Pecij y col. evaluaron los niveles de PCR en 50 pacientes con periodontitis de moderada a severa, y 25 pacientes sanos periodontal y sistémicamente como controles, observando que los pacientes con periodontitis presentaban mayores niveles de PCR en sangre (Peicic et al., 2011, 407). Gomes-Filho y col. reportaron resultados similares con una muestra mayor (359 pacientes) concluyendo que existe una relación entre la EP y los niveles elevados de PCR (Gomes-Filho et al., 2011, 969). Estos estudios concuerdan con lo observado en el presente estudio, en donde los pacientes con EP presentaron un promedio de PCR mayor que en el grupo control, dicha diferencia fue estadísticamente significativa en contraste con el grupo control, donde ninguno de los pacientes mostró alteración de los parámetros normales de PCR.

Katagiri y col. sugieren que la PCR puede desarrollar un papel independiente en la reducción de la hemoglobina glucosilada en pacientes con DM2 que recibieron tratamiento periodontal de raspado y alisado, combinado con antibioticoterapia (Katagiri et al., 2009, 308). Por otro lado, Koromantzos y col. no encontraron diferencias en los niveles de PCR de los pacientes con periodontitis moderada a avanzada y con DM2 no controlada que fueron sometidos a tratamiento periodontal no quirúrgico; por el contrario, se observó una mejoría clínica y un efecto estadísticamente significativo en el control glicémico (Koromantzos et al., 2012, 3). En nuestro estudio, se observó que los pacientes con EP/DM2 presentan una PI de $2.56 \mathrm{~mm}$ así como PCR de $5.2 \mathrm{mg} / \mathrm{L}$, que es más baja que la observada en los pacientes con EP únicamente (PI= 3.14, $\mathrm{PCR}=5.9)$.

Se puede considerar a la PCR como un importante indicador de estados inflamatorios, más notable cuando se trata de cuadros inflamatorios agudos; sin embargo, es posible encontrar alteraciones de sus niveles en estados crónicos. Está bien descrito que la patogenia de la enfermedad periodontal es un proceso complejo, donde la infección bacteriana y la inflamación juegan un papel de suma importancia. Los procesos infecciosos que se dan en la periodontitis estimulan la producción de diversos mediadores inflamatorios entre los cuales se encuentra el TNF- 
$\alpha$ e IL-6, que tienen la capacidad de estimular a las células hepáticas para que produzcan PCR. Esto explica no solo que los pacientes con enfermedad periodontal presenten niveles elevados de PCR en sangre, sino que dichos niveles se incrementen de manera notable en pacientes con EP severa (Arroniz et al., 2013, 1026). En el presente estudio, los valores más elevados de PCR en el grupo con EP se pueden explicar debido a que se presenta una mayor PI en este grupo, y esto se puede traducir a una mayor destrucción de los tejidos periodontales con elevados niveles de inflamación en los sitios enfermos. Por otro lado, el grupo con EP+DM fue el que presentó un mayor descontrol glicémico, y como está reportado, existe una relación directa entre el descontrol glicémico y una enfermedad periodontal más destructiva. Al no controlar la enfermedad periodontal, los niveles séricos de glucosa en sangre, así como su control, son más complicados, lo que conlleva a una glicemia elevada.

Goyal y col. reportaron una relación positiva entre la EP y la PCR; así mismo, reportaron que los niveles de PCR se encontraron más elevados en pacientes jóvenes con periodontitis agresiva (Goyal et al., 2014, 484). De igual forma Kanaparthy y col. encontraron una relación directamente proporcional entre la severidad de la enfermedad y la concentración de niveles séricos de PCR (Kanaparthy et al., 2012, 261). En el presente estudio, concordamos con estos resultados, ya que se mostró que valores mayores de PCR se correlacionan con el aumento de la PI, lo que se puede relacionar con estadios más severos de EP.

Pradeep y col. establecieron 3 grupos de estudio (controles sanos, sujetos con gingivitis y sujetos con periodontitis) buscando relacionar los niveles de PCR en sangre y en el líquido crevicular, observando niveles mas elevados de PCR en el grupo de pacientes con EP tanto en sangre como en liquido gingival crevicular (Pradeep et al., 2010, 133). Kanaparthy y col. reportaron una significancia $(\mathrm{P}=0.012)$ en los resultados sobre niveles mayores de $\mathrm{PCR}$ en grupos de periodontitis tanto crónica como agresiva, comparadas con el grupo control, y entre los grupos de periodontitis crónica y periodontitis agresiva, siendo esta última el grupo con niveles más altos, concordando con los resultados de nuestro estudio ( $\mathrm{P}<0.001)$ (Kanaparthy et al., 2012, 261).

Por otro lado, existen diferencias entre la concentración de PCR y el IMC de los 3 grupos: a mayor IMC mayor es la concentración de la PCR; por el contrario, la pérdida de peso disminuye el valor de la PCR. Los valores de PCR también se asocian con muchas características de la resistencia a la insulina o síndrome metabólico, incluyendo la DM. La producción de PCR predice el desarrollo de la DM2, independientemente de los factores de riesgo tradicionales. En los 
individuos obesos propensos a la resistencia a la insulina, los valores elevados de PCR caen en paralelo con las mejoras en la resistencia a la insulina que están asociados con la pérdida de peso, pero la asociación entre la PCR y la resistencia a la insulina es independiente al IMC. Del mismo modo, el ejercicio físico y el consumo moderado de alcohol están asociados con la reducción de la concentración de PCR de línea de base. Por el contrario, la asociación positiva de los valores de PCR con otros factores de riesgo cardiovascular clásicos, como la enfermedad periodontal y el tabaquismo, parece más claramente relacionada con la inflamación arterial local (Swift et al., 2012, 1028).

\section{Conclusión}

Se encontraron diferencias estadísticas significativas al comparar la concentración de PCR en los 3 grupos que se compararon, siendo el grupo de pacientes sanos el que presentaba el menor promedio y los mayores promedios para los grupos de EP y EP/DM2. Aunque se notó esta diferencia, existen diversos factores tanto locales como sistémicos que pueden potencialmente influir en los niveles de PCR y estos representan una limitación y dificultad al momento de interpretar las pruebas. Por lo que su uso como marcador inflamatorio debe ser cuidadoso y en todo caso descartar aquellas condiciones que pudieran modificar su expresión.

\section{Referencias}

American Diabetes Association. (2010). Diagnosis and Classification of Diabetes Mellitus. Diabetes Care. 33: S62-S69.

Aramesh S, Nelson R, Tulloch-Reid, Hanson R, Sievers M, Taylor G. (2005). Periodontal disease and mortality in type 2 diabetes. Diabetes Care. 28: 27-32. 
Arróniz PS, Furuya MA, Gómez MA, Garzán TJ, Redondo CC, Martínez LJA. (2013). Proteína C reactiva de alta especificidad como marcador de la enfermedad periodontal. Oral. 14: 10261029.

Bulut U, Develioglu H, Taner IL, Berker E. Interleukin 1 beta levels in gingival crevicular fluid in type 2 diabetes mellitus and adult periodontitis. (2001). Journal of Oral Science. 43: 171177.

Cekici A, Kantarci A, Hasturk H, Van Dyke T. (2014). Inflammatory and immune pathways in the pathogenesis of periodontal disease. Periodontology 2000. 64: 57-80.

Flegal K, Kit B, Orpana H, Graubard B. (2013). Association of all-cause mortality with overweight and obesity using standard body mass index categories. Journal of American Medical Association. 309: 71-82.

Gomes-Filho IS, Freitas J, Seixas S. (2011). Chronic Periodontitis and C-Reactive Protein Levels. Journal of Periodontology. 82: 969-978.

Goyal L, Bey A, Gupta ND, Sharma VK. (2014). Comparative evaluation of serum C-reactive protein levels in chronic and aggressive periodontitis patients and association with periodontal disease severity. Contemporary Clinical Dentistry. 5: 484-488. 
Kanaparthy A, Kanaparthy R, Niranjan N. (2012). Evaluation of serum C reactive protein levels in subjects with aggressive and chronic periodontitis and comparison with healthy controls. Dental Research Journal. 9: 261-265.

Katagiri S, Nitta H. Nagasawa T. Uchimura I. Izumiyama H. Inagaki K, Kikuchi T, Noguchi T. Kanazawa M. Matsuo A. Chiba H. Nakamura N. Kanamura N. Inue S. Ishikawa I. Izumi Y. (2009). Diabetes Research and Clinical Practice. 83:308-315.

Kejriwal S, Bhandary R, Thomas B, Kumar S. (2014). Estimation of levels of salivary mucin, amylase and total protein in gingivitis and chronic periodontitis patients. Journal of Clinical and Diagnostic Research. 8: 56-60.

Kinane D. Causas y patogenia de la enfermedad periodontal. Periodontol 2000 (Ed Esp) 2009; 25: $8-20$.

Koromantzos P, Makrilakis K, Dereka X, Offenbacher S, Katsilambros N, Vrotsos IA, Madianos PN. (2012) Effect of non-surgical periodontal therapy on C-Reactive Protein, oxidative stress, and matrix metalloproteinase (MMP)-9 and MMP-2 levels in patients whith type 2 diabetes: A randomized control study. Jpurnal of Periodontology.83:3-10.

Loe H. Periodontal disease. (1993). The sixth complication of diabetes. Diabetes Care. 16: 329334. 
Medzhitov R. Recognition of microorganisms and activatin of the immune response mature. (2007). Nature. 449: 819-826.

Pejcic A. Kesic L.J. MIlasin J. (2011). C-reactive protein as a systemic marker of inflammation in periodontitis. European Journal of Clinical Microbiological \& Infection Disease. 30: 407414.

Peña-Sisto M, Peña-Sisto L, Díaz-Felizola A, Torres-Keiruz D, Lao-Salas N. La enfermedad periodontal como riesgo de enfermedades sistémicas. (2008). Revista Cubana de Estomatología. 45: 1-9.

Pradeep A.R, Shiva-Manjunath R.G, Kathariya R. (2010). Progressive periodontal disease has a simultaneous incremental elevation of gingival crevicular fluid and serum CRP levels, Journal of Investigative and Clinical Dentistry. 1: 133-138.

Preshaw P, Taylor J. (2011). How has research into cytokine interactions and they role in driving immune responses impacted our understanding of periodontitis. Journal of Clinical Periodontolology. 38: 60-84.

Swift D, Johannsen N, Earnest C, Blair S, Church T. (2012). The effect of exercite training modality of C-reactive protein in type 2 diabetes. Medicine and Science in Sports Exercise. 44: $1028-1036$. 
Timpson NJ, Nosesgaard DC, Harbord RH, Zacho J, Frayling TM, Tybjaer-Hansen A, DaveySmith G. (2011). C-Protein Reactive levels and Body Mas Index: elucidating direction of causation through reciprocal Mendelian randomization. International Journal of Obesity. 35: 300-308.

Tsalik EP, Jaggers B, Glickman S, Langley R, Velkinburgh JC. (2012). Discriminative value of inflammatory biomarkers. Journal of Emergency Medicine. 43: 97-106

Wolf D, Lamster B. Contemporary concepts in the diagnosis of periodontal disease. (2011). Dental Clinics of North America. 55: 47-61. 